JÉSSICA LIMBERGER*

ILANA ANDRETTA**

Universidade do Vale do Rio dos Sinos - UNISINOS

(São Leopoldo, Brasil)

\title{
Novas problemáticas sociais: 0 uso do crack em mulheres e a perspectiva de gênero ${ }^{* * *}$
}

\author{
Nuevos problemas sociales: el consumo de crack en las \\ mujeres y la perspectiva de género
}

New social problems: the use of crack in women and the gender perspective

\footnotetext{
* Mestranda em Psicologia Clínica na Universidade do Vale do Rio dos Sinos (UNISINOS), Brasil. Bolsista CAPES/PROSUP. Correio eletrônico: jessica.limberger.psi@gmail.com

** Professora no Programa de Pós Graduação em Psicologia Clínica da Universidade do Vale do Rio dos Sinos (UNISINOS), Brasil. Correio eletrônico: iandretta@unisinos.br

*** Este trabalho faz parte de um estudo maior, intitulado: "Avaliação e Treinamento de Habilidades Sociais em Dependentes Químicos em Tratamento Especializado", do grupo de pesquisa "Intervenções Cognitivo - Comportamentais: Estudo e Pesquisa”, da Universidade do Vale do Rio dos Sinos-UNISINOS, Brasil. Artigo recebeu a 16/02/2015 e aceitado 09/04/2015.
} 


\section{Resumo}

Resumen

Abstract

Objetiva-se compreender o uso do crack em mulheres, sua trajetória de vida e características clínicas, através da perspectiva de gênero. Trata-se de um estudo de caso, de caráter qualitativo. A participante Maria, gestante usuária de crack, encontrava-se em internação hospitalar. Sua história foi permeada por violências, vulnerabilidades e dificuldades familiares. Através dos instrumentos (entrevistas semiestruturada e clínica e Screening Cognitivo), evidenciou-se funções cognitivas preservadas, transtorno de ansiedade generalizada, e transtorno de personalidade antissocial. O preconceito dificultou o acesso ao tratamento, havendo necessidade de intervenções de caráter social, com tratamentos específicos para mulheres usuárias de crack.

PALAVRAS-CHAVE:

Gênero | cocaína crack | mulheres | preconceito | vulnerabilidade

El objetivo de este trabajo es entender el consumo de crack en las mujeres, su historia de vida y las características clínicas, mediante la perspectiva de género. Este es un estudio de caso de carácter cualitativo. La participante María, consumidora de crack embarazada, estaba internada en un hospital. La violencia, las vulnerabilidades y las dificultades familiares forman parte de su historia. Através de los instrumentos (entrevistas semiestructuradas y clínicas y cribado cognitivo), se revelaron una funciones cognitivas preservadas, trastorno de ansiedad generalizada, episodio (hipo)maníaco pasado y trastorno de personalidad antisocial. El prejuicio dificultó el acceso al tratamiento, que requiere intervenciones de carácter social, con tratamientos específicos para las mujeres que usan crack.

\section{PALABRAS CLAVE:}

Género | cocaína crack | mujeres | prejuicio | vulnerabilidad

The objective of this work is to understand the use of crack in women, their life stories and clinical characteristics from a gender perspective. This is a case study of qualitative character. The participant Maria, a pregnant crack user, was in hospital. Her story was permeated by 
violence, vulnerabilities and family difficulties. Through the instruments (semi-structured interviews and clinical and Cognitive Screening), revealed a preserved cognitive functions, generalized anxiety disorder, episode (hypo)maniac past, and antisocial personality disorder. Prejudice made the access to treatment difficult, requiring social character interventions, with specific treatments for women who use crack.

\section{KEYWORDS:}

Gender | crack cocaine | women | prejudice | vulnerability 


\section{Introdução}

O crack configura-se como uma droga de grande impacto, indo além dos danos específicos ao organismo (Kessler \& Pechansky, 2008). Trata-se de um estimulante da atividade cerebral que aumenta a atividade de sistemas neuronais e gera o estado de alerta exagerado, a insônia e a aceleração dos processos psíquicos (Alves, Ribeiro, \& Castro, 2011). Inicialmente, o crack foi identificado nos Estados Unidos na década de 1980, principalmente em comunidades que se encontravam em situação de vulnerabilidade social (Bastos \& Bertoni, 2014). Aproximadamente dez anos depois, há relatos sobre o uso crack na população brasileira, que refletem a preocupação de pesquisadores e profissionais da saúde sobre o uso e as consequências dessa droga (Kessler \& Pechansky, 2008).

Atualmente, o uso de crack no Brasil torna-se uma realidade grave, repercutindo na sociedade em suas diversas esferas: econômica, educativa, produtiva, afetiva, de saúde e de relações sociais (Cantarelli, Marchesan, Amaral, \& Lemos, 2014). O Brasil é o maior mercado de crack do mundo e o segundo maior mercado de cocaína, representando $20 \%$ do consumo mundial (Laranjeira, 2014). Estima-se que há um milhão de usuários de drogas ilícitas em geral (excetuada a maconha) e que usuários de crack e/ou similares correspondem a $35 \%$ dos consumidores de drogas nas capitais do país (Bastos \& Bertoni, 2014).

De acordo com o Manual Diagnóstico e Estatístico de Transtornos Mentais - DSM-5 (American Psychiatric Association [APA], 2014), o Transtorno por Uso de Substâncias é definido como um padrão patológico de comportamentos relacionados ao uso contínuo de substância, o qual é mantido apesar dos significativos problemas que traz. Caracteriza-se por um conjunto de sintomas cognitivos, comportamentais e fisiológicos, com alterações nos circuitos cerebrais que podem prosseguir depois da desintoxicação, principalmente em indivíduos com transtornos graves (APA, 2014). Cabe lembrar que o uso de substâncias psicoativas não ocorre apenas em nível neurobiológico, mas principalmente através da estruturação de vínculos sociais e rotinas voltadas ao consumo, pressionando o indivíduo para o uso contínuo, em detrimento de outros campos da vida (Sakiyama, Ribeiro, \& Padin, 2012).

Dados da Pesquisa Nacional sobre o uso do crack apontam que aproximadamente 10\% dos usuários de crack e/ou similares utilizaram algum serviço de internação para tratamento de dependência química nos trinta dias anteriores à pesquisa (Bastos \& Bertoni, 2014). Estudos brasileiros sobre usuários de crack em internação hospitalar relatam que tais pacientes já passaram por tratamentos anteriores, com dificuldade de acesso à internação e recaídas (Gabatz, Schmidt, Terra, Padoin, Silva, \& Lacchini, 2013; Pedroso, Kessler, \& Pechansky, 2013), destacando-se o fato de que a maioria desses constitui-se de homens (Dias, Araújo, \& Laranjeira, 2011), sem diferir de forma significativa com relação à classe econômica è̀ intensidade e à frequência de uso do crack (Freire, Santos, 
Bortolini, Moraes, \& Oliveira, 2012), com dificuldades familiares (Horta, Vieira, Balbinot, Oliveira, Poletto, \& Teixeira, 2014; Moura, Benzano, Pechansky, \& Kessler, 2014; Pettenon, Kessler, Guimarães, Pedroso, Hauck, \& Pechansky, 2014) e funções cognitivas preservadas (Sayago, Lucena-Santos, Horta, \& Oliveira, 2014).

Diante de tais dados, ressaltam-se os estudos acerca do perfil clínico dos usuários de crack, pois são variáveis que necessitam serem contempladas no tratamento. Em um estudo brasileiro com 57 usuários de drogas, identificou-se que os usuários de cocaína e crack apresentavam como comorbidades transtorno depressivo maior; risco de suicídio; episódio hipomaníaco; transtorno de ansiedade generalizada, entre outros (Hess, Almeida, \& Moraes, 2012). Em outro estudo brasileiro, identificou-se em 83,9\% (n=31) outro transtorno além do Transtorno por Uso de Substâncias. Aqueles que utilizavam cocaína/crack preencheram critérios diagnósticos para Transtorno Psicótico Induzido por Substância; Esquizofrenia; Transtorno Disfórico Pré-Menstrual; Transtorno de Déficit de Atenção/Hiperatividade e Transtorno de Personalidade Antissocial (Silva, Kolling, Carvalho, Cunha, \& Kristensen, 2009).

Os usuários de crack apresentam maiores taxas de Transtorno de Personalidade Anti-Social (Kessler, Terra, Faller, Stolf, Peuker, \& Pechansky, 2012), razão pela qual cometem crimes. Um estudo brasileiro evidenciou que ser usuário de drogas não determina o comportamento criminoso, mas sim o Transtorno de Personalidade Antissocial (Oliveira, 2010). Tal transtorno constitui um padrão invasivo de desrespeito e violação dos direitos, iniciando na infância ou adolescência e continua na idade adulta (APA, 2002). Além disso, caracteriza-se pela impulsividade, expressa na dificuldade de planejar o futuro, através de escolhas que tragam a satisfação imediata e desconsideram as consequências e leis (Del-Bem, 2005). Desta forma, os tratamentos necessitam contemplar tanto o Transtorno por Uso de Substâncias, como o de Personalidade Anti-Social (Bordin \& Offord, 200o). Sabe-se que tais tratamentos representam desafios, mas são possíveis de acordo com a motivação dos indivíduos para a mudança e a aliança terapêutica estabelecida (Costa \& Valerio, 2008).

Percebe-se que o uso de substâncias é complexo e requer respostas a esta demanda social. Entretanto, por muito tempo a resposta da sociedade foi o preconceito, deixando de atentar para as necessidades e especificidades dos usuários de drogas (Sodelli, 2011). No que diz respeito às mulheres usuárias de drogas, o preconceito torna-se ainda maior, pois a imagem historicamente construída da mulher, como mãe, cuidadora e esposa, não condiz com a imagem da mulher usuária de droga e/ou participante do narcotráfico (Souza, Oliveira, \& Nascimento, 2014).

Em uma revisão sistemática de estudos nacionais e internacionais de 2003 a 2013, sobre consumo de crack, gênero e vulnerabilidade, evidenciou-se que as produções priorizam a masculinidade e a utilizaram como referencial para a compreensão do uso de drogas e da sexualidade feminina (Ramiro, Padovani, \& Tucci, 2014). Com isso, a 
compreensão do uso de drogas no âmbito da saúde, especificamente da saúde da mulher, necessita ir além das dimensões biomédicas, buscando um olhar mais amplo sobre o processo saúde/doença, que contemple as especificidades da mulher enquanto sujeito social (Souza, Oliveira, \& Nascimento, 2014).

A construção dos gêneros masculino e feminino repercute na maneira de perceber as condutas de homens e mulheres em comportamentos de riscos e transgressões (Oliveira, Paiva, \& Valente, 2007). Ao consumir o crack, as mulheres são rotuladas como irresponsáveis, prostitutas e criminosas. Tais rótulos não condizem com os comportamentos culturalmente esperados, que são vistas como sexo dócil e frágil (Cruz, Oliveira, Coimbra, Kantorski, Pinho, \& Oliveira, 2014). A discriminações de gênero no contexto brasileiro repercutem também nas representações sociais de homens usuários de crack, que percebem as mulheres usuárias de crack como um "corpo feio", que "não pegariam" e exibem as suas cicatrizes decorrentes do uso do crack como sinônimo de virilidade (Romanini \& Roso, 2014).

As diferenças de gênero expressam-se nas motivações de uso, nas consequências psicológicas das substâncias, diferenças metabólicas, comorbidades físicas e psiquiátricas. Soma-se a isso os fatores socioculturais e genéticos e as implicações na gestação e amamentação (Wolle \& Ziberman, 2011). A intensidade do abuso é maior nas mulheres, que relatam consumir 21 pedras de crack em dias de maior uso, enquanto homens relatam consumir 13 pedras (Bastos \& Bertoni, 2014). Tal intensidade já havia sido evidenciada há uma década, quando $87 \%$ dos usuários de cocaína em suas diversas formas (inalada, fumada, injetada) eram homens, e daqueles que a utilizavam em maior quantidade, $80 \%$ eram mulheres (Orsi, Kessler, Pechansky, Araújo, Oliveira, \& Souza, 2004). Dados da Pesquisa Nacional sobre o uso de crack apontam que 29,9\% das mulheres dependentes de crack e similares já se prostituíram para obter a droga, enquanto essa proporção foi de 1,3\% entre os homens. Mais de metade das usuárias engravidaram ao menos uma vez desde o início do uso do crack/similares, configurando riscos na gestação e no desenvolvimento neurológico e intelectual das crianças expostas ao uso da droga. Das mulheres, $44,5 \%$ relataram já ter sofrido violência sexual na vida, contra $7,0 \%$ relatos de mesma natureza em homens (Bastos \& Bertoni, 2014).

Estudos sobre mulheres usuárias de crack apontam para o início do uso de drogas ainda na adolescência (Marangoni \& Oliveira, 2013), com uso atual de outras drogas concomitantemente (Costa, Soibelman, Zanchet, Costa, \& Salgado, 2011) a comorbidades psiquiátricas (Lima, Macedo, Braga, Lemos, \& Silva Júnior, 2011) e a níveis séricos elevados de alumínio (Pechansky, Kessler, Von Diemen, Bumaguin, Surratt, \& Inciardi, 2007). A prostituição é apontada pelas mulheres como uma forma de obtenção do crack (Pedroso, Kessler, \& Pechansky, 2013), acarretando doenças sexualmente transmissíveis, HIV e AIDS (Correa, Matumoto, \& Lonardi, 2008; Malta, et al., 2008; Nappo, Sanchez, 
\& Oliveira, 2011; Pinto, Tancredi, Buchalla, \& Miranda, 2014; Von Diemen, De Boni, Kessler, Benzano, \& Pechansky, 2010).

A exposição a eventos traumáticos durante a infância e a adolescência (agressões e abusos físicos), além de transtorno de estresse pós-traumático têm sido questões abordadas em diversos estudos com mulheres usuárias de crack (Grassi-Oliveira, Pezzi, Daruy-Filho, Viola, Francke, Leite, \& Brietzke, 2012; Tractenberg, Viola, Rosa, Donati, Francke, Pezzi, \& Grassi-Oliveira, 2012; Viola, Tractenberg, Pezzi, Kristensen, \& Grassi-Oliveira, 2013). Dessa forma, maus-tratos na infância constituem fatores de risco para o início do uso do crack em mulheres, o que vale também para a convivência com familiares usuários e para a baixa escolaridade.

Dessa forma, há desafios ao poder público brasileiro, principalmente aos serviços de saúde, segurança pública e assistência social (Bastos \& Bertoni, 2014). Nesse sentido, o Ministério da Saúde, através do Plano Integrado de Enfrentamento ao Crack e outras Drogas amplia as redes de atenção à saúde e de assistência social para usuários de crack e outras drogas (Brasil, 2010). Dentre os Pontos de Atenção propostos pela Rede de Atenção Psicossocial, há unidades de internação em hospitais gerais, através de internações de curta duração - de uma a três semanas (Brasil, 2011). Assim, os hospitais gerais contribuem na estruturação de uma rede de atenção integral em saúde mental no Sistema Único de Saúde, inserindo-se na rede de cuidados como uma alternativa terapêutica viável em diversos municípios (Anzanello, Dias, Dalmolin, Monteiro, \& Oliveira Neto, 2013).

Tendo em vista os aspectos apresentados, percebem-se avanços de estudos na caracterização dos usuários; entretanto, os aspectos culturais e sociais também necessitam ser contemplados (Jorge, Quinderé, \& Yasui, 2013), havendo carência de produções científicas acerca das vulnerabilidades e da perspectiva de gênero que permeiam o uso do crack (Ramiro, Padovani, \& Tucci, 2014). Desta forma, objetiva-se compreender o uso do crack em mulheres, sua trajetória de vida e características clínicas, através da perspectiva de gênero.

\section{Metodologia}

Trata-se de uma pesquisa qualitativa, de estudo de caso único, proporcionando um aprofundamento do fenômeno no contexto social em que está inserido (Yin, 2001). Salienta-se que este estudo é um recorte de uma pesquisa maior, intitulada: "Avaliação e Treinamento de Habilidade Sociais em Dependentes Químicos em Unidades Especializadas", aprovada pelo Comitê de Ética em Pesquisa da Universidade do Vale do Rio dos Sinos (UNISINOS), sob parecer $n^{\circ} 13.172$.

A participante deste estudo foi escolhida intencionalmente, por estar grávida no momento do tratamento do uso do crack. Foram excluídas participantes com síndrome 
psicótica e prejuízos cognitivos, pois em tais circunstâncias há comprometimento na visão de realidade e na assimilação das informações (APA, 2014).

No que diz respeito aos instrumentos, utilizou-se uma entrevista semiestruturada, desenvolvida pelo grupo de pesquisa "Intervenções Cognitivo Comportamentais: Ensino e Pesquisa", no qual as autoras são integrantes. A entrevista é composta por dados sociodemográficos, informações familiares e sobre o uso de drogas, além dos itens do Manual Diagnóstico e Estatístico de Transtornos Mentais - DSM-5 (APA, 2014) para diagnóstico de Transtorno por Uso de Substâncias. O Screening Cognitivo do WAIS-III (Wechsler, 2012) foi utilizado para avaliar as funções cognitivas.

A Régua de Prontidão para a Mudança também foi utilizada, pois trata-se de uma técnica analógico-visual, que busca identificar, a partir da visão do paciente, em qual estágio de mudança ele se encontra. A régua é composta por números de $1 \mathrm{a} 10$, sendo 1 "De modo algum pronto para mudar" e 10: "Trabalhando ativamente na mudança ou mantendo a mudança”. O avaliador pergunta ao paciente sobre quais dos pontos da régua correspondem com o quanto ele está pronto no presente momento, para mudar seu comportamento (Velásquez, Maurer, Crouch, \& DiClemente, 2001). As entrevistas clínicas "Mini International Neuropsychiatric Interview Plus" (MINI-PLUS) e "Structured Clinical Interview for DSM Disorders" (SCID-II) foram utilizadas para avaliar, a partir dos critérios diagnósticos do Manual Diagnóstico e Estatístico - DSM-IV-TR (APA, 2002), os principais transtornos psicóticos e do humor e os seguintes transtornos de personalidade: histriônico, narcisista, borderline e antissocial.

O local de coleta de dados foi escolhido por conveniência, sendo um hospital geral de uma cidade de pequeno porte, no norte do estado do Rio Grande do Sul (Brasil), que dispunha de leitos de internação para a saúde mental. A participante do estudo foi contatada após a autorização do hospital, mediante Carta de Anuência. O processo de consentimento livre e esclarecido deu-se através da explicação dos objetivos e procedimentos da pesquisa, assegurando a voluntariedade no estudo, o sigilo dos dados e o anonimato, deixando claro que a participante poderia desistir a qualquer momento, sem nenhum prejuízo. Por fim, o Termo de Consentimento Livre e Esclarecido (TCLE) foi lido em conjunto com a participante, que concordou em participar do estudo, assinando duas vias do TCLE, ficando uma via com a participante e outra via com a pesquisadora. A devolução dos resultados da pesquisa foi oferecida à participante e ao hospital.

Os dados foram coletados pela primeira autora, tendo em vista que os principais protagonistas da pesquisa qualitativa são o pesquisador e suas relações com o sujeito pesquisado, que é reconhecido em sua singularidade (Rey, 2002). O contato foi realizado em uma sala do hospital, possibilitando um ambiente de sigilo entre a pesquisadora e a participante, sem a interferência de externos. Os instrumentos foram aplicados no oitavo dia de internação, em dois momentos, a fim de evitar a fadiga de maneira a não interferir nas rotinas do hospital. No turno da manhã foi aplicada a entrevista semi-estruturada, 
a Régua de Prontidão para a Mudança e a MINI-Plus. No turno da tarde, foi aplicado o Screening Cognitivo do WAIS-III e a SCID-II.

A análise dos dados deu-se a partir de uma perspectiva interpretativa, que enfatiza o significado das ações, onde o pesquisador constrói o conhecimento a partir das experiências do participante sobre o fenômeno estudado. Desta forma, novos conhecimentos são gerados a partir dos conhecimentos e pressupostos revistos, possibilitando um entendimento em profundidade (Turato, 2005).

\section{Resultados e Discussão}

Maria, uma jovem negra de 25 anos, estava internada em um hospital geral do norte do Rio Grande do Sul (Brasil) devido o uso de crack. Ao ser convidada a participar da pesquisa, Maria mostrou-se disposta para contar sobre sua história, falando com clareza e ouvindo as perguntas com atenção.

Maria nasceu em uma cidade pequena, no estado do Rio Grande do Sul. Sua mãe era doméstica e teve pouco contato com o seu pai na infância, recordando-se apenas de duas vezes em que falou com o mesmo e posteriormente não o viu, pois o pai nunca mais a visitou. Questionada se procurou o pai depois deste período, Maria refere que não quis procurá-lo, pois "ele é que deveria ter procurado".

Durante a infância, Maria conviveu com seus cinco irmãos, sendo a irmã mais nova. Atualmente tem pouco contato com as duas irmãs, e os dois irmãos encontram-se presos por crimes relacionados ao uso de drogas. Desde pequena, sua mãe tinha problemas com o uso do álcool e batia em Maria "por qualquer coisa", principalmente quando estava bêbada. A falta da atenção por parte de sua mãe fica evidente na frase "minha mãe só dava atenção para o macho dela", ao se referir ao padrasto.

As dificuldades familiares são apontadas por usuários de drogas em diversos estudos, demonstrando a necessidade de compreensão para além do ponto de vista individual (Horta, Vieira, Balbinot, Oliveira, Poletto, \& Teixeira, 2014; Moura, Benzano, Pechansky, \& Kessler, 2014; Pettenon, Kessler, Guimarães, Pedroso, Hauck, \& Pechansky, 2014). O contexto familiar de Maria em sua infância e adolescência caracterizava-se por repetidas brigas e discussões, dificultando o estabelecimento de vínculos afetivos positivos. Maria recorda que quando via a mãe alterada devido ao uso do álcool, sempre que possível dirigia-se a casa de sua avó materna, que morava próximo e a acolhia. Assim, a presença da avó foi um fator de proteção, na medida em que proporcionou amparo, evitando riscos maiores.

Ao ser questionada sobre a opinião dos familiares sobre o seu uso de drogas, a mãe de Maria acredita que "foi culpa das más companhias". Sua mãe chorava ao ver a filha usando drogas e rezava pedindo a Deus para que Maria parasse de utilizar drogas. De 
acordo com o Levantamento Nacional de Famílias dos Dependentes Químicos, na opinião do familiar, o que levou o usuário a usar álcool/drogas foi, em $46,8 \%$ dos casos por andar com más companhias, $26,1 \%$ devido a baixa autoestima, $25,9 \%$ devido a outras pessoas da família ou da escola também usarem, $22,7 \%$ pela ausência do pai, $18,3 \%$ para se enturmar/se soltar, $11,1 \%$ por ser revoltado/indisciplinado e 10,3\% por ser genético, nascer assim. Além disso, dos 3.164 familiares de pacientes em tratamento, 61, 6\% dos usuários possuíam outros familiares usuários, sendo que 57,6\% pertenciam ao núcleo familiar (Brasil, 2013). Os dados de tal pesquisa assemelham-se aos da trajetória de Maria, onde sua mãe e seus irmãos também possuíam problemas com o uso de drogas.

A ausência da função paterna na vida de Maria, fez com que sua mãe tivesse dificuldades em posicionar-se quanto ao uso das drogas, tanto em relação a Maria, como em relação ao irmãos, que possuíam problemas com o uso de drogas. Maria diz que sua mãe custou a perceber que Maria estava usando drogas, e que tinham dificuldades de conversar a respeito. Além disso, o fato de a mãe de Maria possuir problemas com o uso do álcool contribuiu para que o relacionamento entre mãe e filha fosse conturbado.

O início do uso de drogas ocorreu precocemente na vida de Maria. Aos 10 anos, experimentou o cigarro e seguiu usando escondida, pois via a sua mãe fumando todos os dias. Ela recorda que ao ver a mãe fumando, sentiu vontade de fumar também e começou a fumar escondido. Percebe-se que as práticas culturais familiares podem contribuir na experimentação, bem como na continuidade do uso de drogas, pois a família configurase como uma geradora de crenças e de expectativas acerca dos papeis sociais: o modo de vida de homens e mulheres, as relações interpessoais e inclusive acerca do uso de drogas lícitas e ilícias (Seleghin, Inoue, Santos, \& Oliveira, 2011).

Aos treze anos, Maria sai de casa e vai morar na casa de amigas, retornando somente seis meses depois, quando o padrasto saiu de casa. Maria não fala sobre seu padrasto, apenas diz que "não prestava". No período em que Maria estava longe de casa, seu irmão passava na casa de suas amigas para saber se Maria estava bem, mas em todo esse tempo sua mãe não a procurou. Novamente percebem-se as dificuldades familiares e a carência de cuidado e amparo na adolescência, período tão importante do ciclo vital. A literatura evidencia que na maioria dos usuários o início do uso de drogas lícitas ocorre na adolescência, em contextos familiares que contribuem para o uso de drogas ilícitas (Dias, Araújo, \& Laranjeira, 2011; Marangoni \& Oliveira, 2013; Seleghin, Inoue, Santos, \& Oliveira, 2011).

Para pertencer a um grupo de amigos, Maria começou a usar álcool e maconha quando deveria estar na escola. Nesse contexto, o uso de drogas associa-se à necessidade de pertencimento, onde a influência de amigos usuários contribui no início do consumo de drogas ilícitas, em um momento de vulnerabilidade e longe da supervisão de adultos (Marangoni \& Oliveira, 2013). A necessidade de pertencimento ao grupo é inerente à adolescência. Entretanto, Como Maria era constantemente chamada de "nega gorda" 
na escola, teve dificuldades no fortalecimento de um vínculo salutar de amizades. Ela conta que o jeito que conseguia para lidar com essa situação era constantemente se envolvendo em brigas, agindo cada vez mais com impulsividade e agressividade. Com tais comportamentos, acabou aproximando-se de um grupo que faltava as aulas e fazia uso de drogas, sendo este o grupo que Maria encontrou para sentir-se pertencente e que não a chamavam de "nega gorda", em um momento de vida em que há busca pela afirmação em seu círculo de amizades.

Aos dezesseis anos, Maria parou de estudar, quando estava na sexta série. Percebe-se que houve falhas no ambiente familiar e no espaço escolar, na prevenção ao uso de drogas. Maria refere histórico de reprovações e constantes períodos em que "matava aula". Sabe-se que a escola possui um papel fundamental no desenvolvimento de habilidades de vida, como a autonomia, a tolerância a frustrações, bem como o trabalho em equipe (Limberger, Mella, \& Duarte, 2014). Quando a escola não configura-se como fator de proteção, a evasão escolar acaba dificultando as possibilidades de profissionalização na vida de Maria.

A saída da escola ocorreu devido a gravidez. Maria diz que esta primeira filha foi planejada, quando saiu de casa para ir morar com o seu companheiro. Depois de brigas, Maria voltou para a casa de sua mãe e sua filha ficou morando com os avós paternos, devido ao uso do crack de Maria, aos 19 anos. A experimentação do crack ocorreu através de um cunhado, que era traficante. Percebe-se que o contexto em que Maria se encontrava estava relacionado ao uso de drogas, desde a infância com o álcool e o cigarro por parte da mãe, passando pela maconha no grupo de amigos e ao crack através do cunhado.

Para manter o uso do crack, Maria realizava assaltos e chegou a vender coisas de dentro da casa de sua mãe. Durante um período de dois meses, quando sua mãe a expulsou de casa, trabalhou como profissional do sexo para manter o uso do crack. O uso de preservativos ocorreu na maioria das vezes, e em momentos que não utilizou o preservativo, contraiu sífilis. A complexidade de tal prática, que poderia ter acarretado em outras consequências, como a AIDS, fazem Maria recordar desta situação com tristeza: "não gosto de lembrar daquele tempo, me sentia mal, como um objeto". Tal aspecto foi evidenciado em uma etnografia na cracolândia, em São Paulo, compreendendo que a troca de sexo pelo crack também envolve a troca de poderes e submissões, em relacionamentos corporais abusivos.

Através da Entrevista Clínica Estruturada para Transtornos de Personalidade - SCID-II, verificou-se o transtorno de personalidade antissocial, com as seguintes características anteriores aos 15 anos: iniciava lutas corporais com os colegas - "No colégio eu batia direto!"; mentia e trapaceava as outras pessoas, mentindo para a mãe ao dizer que estava na escola e não estava, e quando recebia dinheiro para comprar roupas, comprava maconha; fugia de casa à noite por períodos extensos, ficando dias e até meses longe de casa. Além disso, Maria gazeava aulas desde os 11 anos de idade. Após os 15 anos, con- 
tinuou mentindo para usar drogas, além de repetidas lutas corporais e agressões físicas para com o marido e quando familiares a deixavam irritada. Aos 18 anos, foi presa por envolvimento em assalto e vendia drogas junto com o companheiro, também usuário de drogas.

Faz-se necessário compreender que o Transtorno de Personalidade Antissocial associado ao Transtorno por Uso de Crack insere-se em um contexto de fracos vínculos primários, em relações familiares conflituosas e ausência paterna. Esses fatores, somados à violência física e psicológica contribuem no desenvolvimento de tais transtornos (Marangoni \& Oliveira, 2013). Compreende-se que os sofrimentos na infância, somados aos problemas sociais, acabam desenvolvendo condutas antissociais que refletem na busca por uma droga de alto poder destrutivo, como o crack (Kessler, 2012).

A partir da entrevista clínica Mini International Neuropsychiatric Interview Plus (MINI-PLUS), evidenciou-se como comorbidades ao Transtorno por Uso de Crack o Transtorno de Ansiedade Generalizada, além de episódio (hipo)maníaco passado. A ansiedade e preocupação excessivas, características do Transtorno de Ansiedade Generalizada, causam sofrimento clinicamente significativo e prejuízos em importantes áreas da vida, havendo maior frequência no sexo feminino (APA, 2014).

Com a aplicação do Screening Cognitivo do WAIS-III (Wechsler, 2012), percebe-se que as funções cognitivas estavam preservadas, ou seja, a inteligência basal, a capacidade de organização visuomotora, a organização visoperceptual na resolução de problemas e a atenção e memória foram classificados com o desempenho médio. Outro estudo brasileiro também apontou que as funções cognitivas estavam preservadas em usuários de crack (Sayago, Lucena-Santos, Horta, \& Oliveira, 2014).

No momento da pesquisa, Maria encontrava-se em sua primeira internação hospitalar devido o uso de drogas. Tal internação foi voluntária, referindo que a motivação para procurar tratamento foi pela filha de 3 anos e pela gestação de 7 meses, além de não conseguir dormir devido o uso de crack. A internação de Maria durou 15 dias, de acordo com o estabelecido no Plano Terapêutico Individual, sendo acompanhada pela mãe nas visitas. Maria também frequenta o Centro de Atendimento Psicossocial (CAPS) e um grupo de "Narcóticos Anônimos" de sua cidade.

Antes da internação, Maria fumava em torno de 10 cigarros por dia, e usava crack 4 dias por semana, utilizando 12 pedras cada vez. Em um dia de maior uso, Maria chegou a usar 15 pedras. Chama a atenção que o uso do crack era visto por Maria como uma maneira de esquecer das suas dificuldades. O período do dia em que mais utilizava crack era durante a noite. Depois de colocar a filha para dormir, usava o crack "para esquecer os problemas". Desta forma, fica evidente que o uso de crack revela dificuldades na resolução de problemas, onde a droga torna-se um meio do indivíduo enfrentar pressões externas, acontecimentos cotidianos e situações interpessoais, em vez de manifestar um comportamento assertivo (Caballo, 2003). 
O momento atual de Maria em seu tratamento demonstra a sua ambivalência entre desenvolver outras estratégias para lidar com suas dificuldades de vida e financeiras ou continuar com o uso do crack. Ao apresentar a régua de prontidão para a mudança, que indica o quanto a pessoa se considera pronta para mudar o seu comportamento em relação a uma droga, ela escolheu o crack. Na régua de prontidão, assinalou o número 5, que indica "Preparando-se para mudar". Para ela, a busca voluntária pela internação no hospital foi um passo muito importante para a mudança, como uma oportunidade de retomar a sua vida. Dentre os períodos de tratamento, talvez o período mais importante seja o da desintoxicação, sendo posteriormente encaminhado para os demais recursos terapêuticos disponíveis (Ribeiro \& Laranjeira, 2012).

Para Maria, a religião que pratica (católica) a ajuda no tratamento, ao encontrar forças para manter-se no mesmo. A necessidade de apoio religioso é relatada por usuários de crack, independente da denominação religiosa (Bastos \& Bertoni, 2014). Sabe-se que fatores como religiosidade e espiritualidade contribuem na recuperação de usuários de drogas, quando comparados a usuários que são tratados exclusivamente através meio médico (Sanchez \& Nappo, 2007).

No que diz respeito ao estado civil, Maria é solteira e diz ter dificuldades nos relacionamentos, que muitas vezes foram com usuários de drogas. As fragilidades na manutenção de laços afetivos foram apontadas no estudo brasileiro de Rosseti et al. (2014), pelo fato de que o uso contínuo do crack dificulta a continuidade de vínculos.

Atualmente Maria mora com a mãe e a filha de 3 anos, em uma casa com apenas duas peças, tendo acesso ao banheiro e a geladeira a partir da casa de sua avó, que mora próximo. As outras filhas, de 8 e 6 anos moram com os avós paternos. Apenas a primeira filha foi planejada, as demais nasceram quando esqueceu-se de tomar as pílulas anticoncepcionais. Maria diz que não planeja ter mais filhos depois deste que está gestando.

Ao compreender o uso do crack na vida de Maria, faz-se necessário considerar sua raça e sua situação socioeconômica. Maria trabalha como doméstica, com renda de 1 salário mínimo. Dados do Perfil sobre o Uso de Crack no Brasil apontam um expressivo predomínio de usuários negros nas cenas de uso, sendo aproximadamente $80 \%$. Somado a tal questão, a baixa escolaridade dificulta a inserção no mercado formal, gerando menor disponibilidade financeira e maior vulnerabilidade social (Seleghin, Marangoni, Marcon, \& Oliveira, 2011). A exclusão da economia formal aumenta o número de mulheres negras segregadas nas favelas, que muitas vezes atuam como "mulas" - transportadores de drogas (Alves, 2014). O acesso aos serviços a mulheres negras e pobres necessita ser repensado. Em um estudo brasileiro com 1.068 mulheres com HIV, das quais 50,7\% eram negras e 49,3\% não-negras, houve diferenças estatisticamente significativas em relação a: escolaridade; renda mensal; oportunidades de atendimento por profissionais da saúde, entre outros (Lopes, Buchalla, \& Ayres, 2007). 
Diante da trajetória de vida de Maria e seu uso do crack, fica evidente a necessidade de acesso aos serviços de saúde de maneira contínua. Além disso, outros aspectos necessitam ser considerados, como o acesso a profissionalização e aos seus direitos de moradia e vida digna, tendo em vista que o uso do crack configura-se como um problema social.

\section{A perspectiva de gênero e o uso de crack}

Ao falar sobre mulheres usuárias de crack, é imprescindível compreender o contexto sociocultural no qual se insere essa discussão. No contexto brasileiro, há avanços nas legislações, como a Lei Maria da Penha (Lei no 11.340/o6), que prevê o aumento das punições contra as agressões sofridas pelas mulheres no contexto doméstico ou familiar (Brasil, 2006) e mais recentemente, a Lei no 13.104/15, que inclui o feminicídio no rol dos crimes hediondos, cuja pena é aumentada de um terço até a metade, de acordo com as especificações. Entretanto, apesar de avanços nas legislações, muitas mulheres brasileiras ainda sofrem com violências psicológicas e desigualdades, devido ao estigma que carregam na sociedade contemporânea (Silva, 2010). Desta forma, a imposição cultural da inferioridade da mulher em relação ao homem gera obstáculos na luta pela igualdade. Tal luta torna-se um passo necessário para o progresso da humanidade, não sendo apenas um assunto de mulheres, mas sim de interpelação entre os gêneros (Delgado \& Zambrano, 2010).

A situação da mulher usuária de crack retrata uma sequência de desigualdades: no ambiente familiar, na escola, nos locais de trabalho e no acesso aos serviços de saúde. O preconceito com a mulher usuária de crack repercute em todos os segmentos sociais, devido a discriminação de gênero presente na sociedade brasileira (Romanini \& Roso, 2014). Muitas vezes, o preconceito aparece em mais de uma forma: no preconceito à mulher, negra, usuária de crack, prostituta e sem a convivência com os filhos.

A mulher é vista culturalmente como "cuidadora" e quando esta se encontra em sofrimento mental e necessita de cuidados, há dificuldade da sociedade de ver quem se esconde atrás do uso do crack: a mulher que foi vítima de violências e a mulher que possui necessidade de um tratamento com um olhar biopsicossocial, rompendo com estigmas e julgamentos. Maria relata que demorou para procurar o atendimento pois foi humilhada constantemente pelos parentes por usar crack, tendo receio de passar novamente por estahumilhação, que relaciona-se com as imagens populares, que associam imediatamente o uso do crack à periculosidade e às zonas marginais da cidade (Medeiros, 2014).

Estudos apontam que mulheres usuárias de drogas demoram mais para procurar o tratamento quando comparadas aos homens, devido a estigmatização e o preconceito que sofrem (Rivadeneyra \& Cabrera, 2010; Souza, Oliveira, \& Nascimento, 2014). Um dos maiores obstáculos que as mulheres enfrentam ao buscar um tratamento para o 
uso de drogas concentra-se no estigma social associado ao uso, pois esse gera conflitos com o papel feminino socialmente esperado (Ramiro, Padovani, \& Tucci, 2014). Além disso, o preconceito e a discriminação por parte dos profissionais da saúde também constituem barreiras na permanência do tratamento (Mendes \& Luis, 2004). Maria diz que possuía dificuldades em entender o que os profissionais da saúde lhe diziam, além de relatar a dificuldade de conseguir uma ficha para ser atendida, mesmo estando grávida. Ela conta com alegria de um dia que um profissional da saúde viu que ela estava na fila, grávida, e que este disse que ela tinha prioridade no atendimento e que iria ser chamada logo em seguida.

Quando se trata dos problemas relacionados ao uso de drogas, estudos demonstram uma maior procura de tratamento por parte dos homens (Ramiro, Padovani, \& Tucci, 2014). Entretanto, isso não significa que mulheres não estejam com problemas relacionados ao uso de drogas, mas que estas estão com dificuldade em acessar os serviços de atendimento. Em um estudo qualitativo sobre o contexto assistencial, percebeu-se que para profissionais que trabalhavam em instituições de saúde, havia menor busca de tratamento por mulheres usuárias de drogas. Todavia, com profissionais que trabalhavam diretamente na comunidade, percebeu-se que o quantitativo de mulheres com problemas relacionados às drogas chegava a superar ao dos homens (Oliveira, Paiva, \& Valente, 2007). Desta forma, é necessário estabelecer pontes entre os locais de tratamento e a comunidade, valorizando as ações dos centros de referência (Bastos, 2012).

A mulher usuária de drogas necessita de um olhar diferenciado dos profissionais da saúde, a fim de que estes atentem para as suas necessidades específicas, dando prioridade à promoção de saúde, auto-estima e reinserção social (Lima, Macedo, Braga, Lemos, \& Silva Júnior, 2011). Sobre as necessidades das mulheres usuárias de crack, Maria diz: "A gente precisa de ajuda. Não adianta xingar, tem que ter alguém para sentar e conversar. Se fosse mais rápido para consultar, eu já estaria internada há mais tempo". Percebe-se que as mulheres usuárias de crack carecem de informações claras sobre os possíveis tratamentos e sobre as drogas, de maneira direta e empática. Conforme apontado no estudo de Rivadeneyra e Cabrera (2010), o conhecimento é um fator importante na busca de tratamento. Além disso, dados do Perfil Nacional sobre os Usuários de Crack demonstram a importância do aprimoramento da referência e contrarreferência das mulheres nos serviços de saúde, considerando suas necessidades específicas em espaços que não sejam discriminadas (Bastos \& Bertoni, 2014).

Diante da realidade imposta pela perspectiva de gênero, é essencial uma discussão articulada com a formação profissional, a fim de superar as barreiras em relação ao cuidado, com foco nas necessidades dessa clientela que está cada vez mais presente (Lima, Macedo, Braga, Lemos, \& Silva Júnior, 2011). Nas falas de Maria, fica evidente as questões culturais implicadas na imagem da mulher sobre o seu uso de crack: "Eu acho feio, me sinto angustiada de estar usando, com vergonha. Quando estou na rua 
parece que todo mundo está olhando, aí me sinto mal. Quando não uso fico de cabeça erguida". Percebe-se que o estigma atribuído às mulheres usuárias de crack e a discriminação dificultam, ainda mais, a busca por serviços de saúde. Assim, há necessidade de inovação nas práticas sociais e de saúde, a fim de que consigam acessar esse público (Ramiro, Padovani, \& Tucci, 2014).

Outro fator a ser considerado no uso de crack em mulheres diz respeito aos filhos. Para Maria, não conviver com dois de seus filhos é uma realidade difícil, mas ela acredita ser melhor para eles estarem com os avós paternos: "Eles estão melhor do que eu, o que eles precisam, eles têm". O cuidado com a filha de 3 anos, que mora com Maria, é partilhado com sua mãe e avó. Na gestação atual, Maria diz que reduziu o uso de drogas, mas não conseguiu parar de usar. Conforme Oliveira e Paiva (2007), condutas como o afastamento dos filhos e redução do consumo de drogas durante a gravidez parece colocá-las, de alguma maneira, mais próximas dos papéis social e culturalmente estabelecidos para as mulheres, na função de mãe e cuidadora.

A gestação de mulheres usuárias de crack requer atenção, considerando os dados do perfil dos usuários de crack e/ou similares no Brasil, que demonstram que mais da metade das usuárias engravidaram ao menos uma vez desde o início do uso do crack/similares. Maria diz que se sente culpada em usar crack durante as gestações, tendo dificuldades em ficar abstinente: "Os meus parentes me xingam, dizem que eu tô fazendo mal para o bebê, mas eu não consigo parar (...). O que eu consigo fazer é usar menos quando eu tô grávida". Desta forma, sendo o cuidado e a educação dos filhos uma função tradicionalmente feminina, quando a mulher negligencia este comportamento passa a ser taxada como egoísta e irresponsável para com os filhos (Oliveira \& Paiva, 2007). Com isso, o uso de drogas em mulheres pode ser subdiagnosticado, pois as gestantes podem prever uma desaprovação dos profissionais da saúde, negando ou relatando um consumo menor de drogas (Kassada, Marcon, Pagliarini, \& Rossi, 2013).

A diminuição do uso de drogas no período da gestação também pode ser compreendida como uma tentativa de mudança de vida, e ao mesmo tempo de trazer um novo sentido à vida. Maria diz: "Se não fosse pelas minhas filhas eu não estava aqui, eu ia me destruir mais ainda nas drogas". Desta forma, assim como a motivação para o tratamento está ligada a gestação, as buscas pela mudança de comportamento em relação às drogas parecem ter amparo nos filhos. Em um estudo sobre a percepção de gestantes sobre o consumo de drogas ilícitas na gestação, as relações entre as mães e seus filhos foram dificultadas devido o uso do crack e outras drogas, ocasionando muitas vezes até a perda de guarda, por vezes sendo encaminhados para a adoção (Portela, Barros, Frota, Landim, Caetano, \& Farias, 2013). Além disso, percebeu-se o despreparo e a falta de conhecimento sobre o uso de drogas e seus efeitos no decorrer da gestação, bem como as consequências para o recém-nascido. As mulheres que sabiam do risco, diziam que 
não conseguiam parar de usar, devido o vício (Portela, Barros, Frota, Landim, Caetano, \& Farias, 2013).

O uso contínuo de crack durante a vida de Maria, por mais de 6 anos, contribuiu para a dificuldade de abstinência durante a gestação. A gravidez não funcionou como "turning point", ou seja, a ocasião significativa da gestação não contribuiu para a interrupção do uso de droga, como evidenciam outros estudos brasileiros (Marangoni \& Oliveira, 2013; Portela, Barros, Frota, Landim, Caetano, \& Farias, 2013). Tratando-se de um problema complexo, o tratamento para a mulher usuária de crack requer um suporte social que muitas vezes não se apresenta para as mulheres, ora pelos companheiros também serem usuários, ora pelos laços familiares estarem fragilizados. Desta forma, sem o suporte no tratamento, as possibilidades de recaída são maiores.

\section{Considerações finais}

O uso do crack em mulheres trata-se de um problema social. Para tanto, as responsabilidades necessitam ser compartilhadas nos diferentes segmentos sociais, perpassando a educação, a saúde e a assistência social.

O estudo de caso de Maria permitiu identificar sequências de vulnerabilidades e de agravos no decorrer de sua vida. Uma série de direitos lhe foi negada: ao deixar de frequentar a escola e não ter um acompanhamento diante dessa situação; a falta de apoio psicológico para lidar com as agressões físicas e problema do uso de álcool de sua mãe, bem como a ausência do pai; a carência de informações claras sobre o uso da droga e suas possibilidades de tratamento.

Percebe-se que o crack acaba mascarando uma série de problemas sociais, que muitas vezes não são vistos e acabam transformando-se em preconceitos. Com o uso do crack, parece que a sociedade não consegue ver a mulher por trás do uso. É como se ela deixasse de ser mulher e passasse a ser "sem-vergonha", "prostituta", "que não cuida dos filhos", "que transmite doenças". Diante de tantos avanços garantidos historicamente aos direitos das mulheres, parece que tais direitos não chegam a alcançar as mulheres usuárias de drogas, e especialmente as mulheres usuárias de crack.

O preconceito agrava ainda mais essa situação de vulnerabilidade, dificultando o acesso ao tratamento. É imprescindivel repensar o acesso ao tratamento e garantir a continuidade do mesmo, partindo das especificidades das mulheres, onde os tratamentos das mulheres deixem de ser embasados em estudos realizados com homens. Urge a necessidade de pesquisas sobre as mulheres usuárias de crack, indo além da caracterização do uso, a fim de permitir que as necessidades das mulheres sejam evidenciadas, e que estratégias de tratamento sejam planejadas e amparadas empiricamente. 
Tratando-se de uma questão de cunho social, ao se pensar em um plano de tratamento ao uso de drogas, torna-se imprescindível considerar outros aspectos que estão relacionados com a saúde. São necessárias condições de vida digna, profissionalização e maior acesso aos direitos. As mulheres necessitam de informações claras e precisas sobre o tratamento e sobre o uso de drogas. A falta de informação não está presente apenas nas mulheres, mas também na sociedade como um todo. É imprescindível que a mulher usuária de crack seja vista como uma mulher que necessita de ajuda e de cuidados específicos.

Repensar os tratamentos de mulheres usuárias de crack não significa negar os tratamentos existentes. Muito pelo contrário, significa potencializar práticas exitosas e contribuir para que as ações sejam cada vez mais próximas das necessidades das mulheres. Levando em consideração os aspectos apresentados, almeja-se que os abismos entre profissionais da saúde e mulheres usuárias de crack sejam transformados em pontes, para uma travessia segura e amparada.

\section{Referências}

ALVES, H. N. P., RIBEIRO, M., \& CASTRO, C. D. (2011). Cocaína e Crack. In: Diehl, A., Cordeiro, D. C., \& Laranjeira, R. (eds.), Dependência Química: prevenção, tratamento e políticas públicas. (pp. 170-179). Porto Alegre: Artmed.

ALVES, J. A., (2014). On Mules and Bodies: Black Captivities in the Brazilian Racial Democracy. Critical Sociology, 1-20. doi: 10.1177/0896920514536590.

AMERICAN PSYCHIATRIC ASSOCIATION. (2002). Manual Diagnóstico e Estatístico de Transtornos Mentais. DSM-IV-TR. Porto Alegre: Artmed.

American Psychiatric Association. (2014). Manual Diagnóstico e Estatístico de Transtornos Mentais. DSM-5. Porto Alegre: Artmed.

AMORIM, P. (2000). Mini International Neuropsychiatric Interview (MINI): validação de entrevista breve para diagnóstico de transtornos mentais. Rev. Bras. Psiquiatr, 22(3), 106-115.

ANZANELLO, J., DIAS, M. T. G., DALMOLIN, B., MONTEIRO, J. R., \& OLIVEIRA NETO, A. B. (2013). Percepção dos usuários de crack e seus familiares quanto ao acesso e serviços oferecidos em hospitais gerais. Saúde em Debate, 37, 92-102. Retrieved from: http://cebes.com.br/site/ wp-content/uploads/2014/o6/RSD_EspecialDrogas_REV2905_Web.pdf

BASTOS, F. I. (2012). Crack: personalidade antissocial é tônica entre usuários. In: Informe ENSP. Retrieved from: http://www.ensp.fiocruz.br/portal-ensp/informe/site/materia/detalhe/31006 
BASTOS, F. I., \& BERTONI, N. (org) (2014). Pesquisa Nacional sobre o uso de crack. Retrieved from http://www.icict.fiocruz.br/sites/www.icict.fiocruz.br/files/Pesquisa\%20Nacional\%2O sobre\%200\%2oUso\%2ode\%2oCrack.pdf

BORDIN, I. A. S., \& OFFORD, D. R. (2000). Transtorno da conduta e comportamento antisocial. Revista Brasileira de Psiquiatria, 22 (2), 12-15. doi:10.159o/S1516-44462000ooo60ooo4.

BRASIL. (2006). Lei no 11.340, de 7 de agosto de 2006. Retrieved from: http://www.planalto.gov. br/ccivil_03/_ato2004-2006/2006/lei/l11340.htm

BRASIL. (2010). Plano Integrado de Enfrentamento ao Crack e outras Drogas. Retrieved from: http://www.planalto.gov.br/ccivil_03/_Ato2007-2010/2010/Decreto/D7179.htm

BRASIL. (2011). Decreto 7.508, de 28 de junho de 2011. Retrieved from: http://www.planalto.gov. br/ccivil_03/_ato2011-2014/2011/decreto/D7508.htm

BRASIL. (2013). LENAD Família. Retrieved from: http://inpad.org.br/_lenad-familia/.

BRASIL. (2015). Lei no 13.104, de 9 de março de 2015. Retrieved from: http://www.planalto.gov. br/ccivil_O3/_Ato2O15-2018/2015/Lei/L13104.htm

CANTARElli, N, D. C., MARChESAN, E. K, R., AMARAL, M. C., \& LEMOS, J. C. (2014). Perfil dos usuários de substâncias psicoativas de um Hospital Universitário no Rio Grande do Sul. Saúde, 4O(1), 85-9o. doi:10.5902/2236583412777

CORREA, N. A. B., MATUMOTO, F. H., \& LONARDI, M. V. C. (2008). Doenças sexualmente transmissíveis em mulheres profissionais do sexo, Umuarama, Estado do Paraná. RBAC, $4 O(3)$, 209-213. Retrieved from: http://www.sbac.org.br/rbac/o12/179.pdf

COSTA, G. M., SOIBELMAN, M., ZANCHET, P. M. C., SALGADO, C. A. I. (2012). Pregnant crack addictsin apsychiatric unit.J.bras.psiquiatr, 61(1).8-12.doi:10.1590/Soo47-20852012000100003

COSTA, J. B. P., \& VALERIO, N. I. (2008). Transtorno de personalidade anti-social e transtornos por uso de substâncias: caracterização, comorbidades e desafios ao tratamento. Temas em Psicologia, 16(1), 119-132. Retrieved from: http://pepsic.bvsalud.org/scielo.php?script=sci arttext\&pid=S1413-389X20080o0100010\&lng=pt\&tlng=pt.

CRESWELL, J. W. (2010). Projeto de Pesquisa: métodos qualitativo, quantitativo e misto. Porto Alegre: Artmed.

CRUZ, V. D., OLIVEIRA, M. M., COIMBRA, V. C. C., KANTORSKI, L. P., PINHO, L. B., \& OLIVEIRA, J. F. (2014). Vivências de mulheres que consomem crack. Revista da Rede de Enfermagem do Nordeste,15(4). 639-649. doi: 10.15253/2175-6783.2014000400011.

DEL-BEM, C. M. (2005). Neurobiologia do transtorno de personalidade anti-social. Revista de Psiquiatria Clínica, 32(1). 27-36. Retrieved from: http://www.hcnet.usp.br/ipq/revista/vol32/ $\mathrm{n} 1 / 27 . \mathrm{html}$ 
DELGADO, X. N. O., \& ZAMBRANO, A. C. D. (2009). Identidad de gênero: ¿obstáculo al desarollo o acceso a la equidad? Revista em Ciencias Sociales, 4, 271-282. Retrieved from: http://www. icesi.edu.co/revistas/index.php/revista_cs/article/view/445/445

DIAS, A. C., ARAÚJO, M. R., \& LARANJEIRA, R. (2011). Evolução do consumo de crack em coorte com histórico de tratamento. Rev Saúde Pública, 45(5), 938-848. Retrieved from http://www. scielo.br/scielo.php?pid=So034-89102011000500016\&script=sci_arttext

FLICK, U. (2004). Uma introdução à pesquisa qualitativa. Porto Alegre: Bookman.

FREIRE, S. D., SANTOS, P. L., BORTOLINI, M., MORAES, J. F. D., \& OLIVEIRA, M. S. (2O12). Intensidade de uso de crack de acordo com a classe econômica de usuários internados na cidade de PortoAlegre/Brasil.J. Bras. Psiquiatr, 61(4), 221-226.doi:10.1590/Soo47-20852012000400005

GABATZ, R. I. B., SCHMIDT, A. C., TERRA, M. G., PADOIN, S. M. M., SILVA, A. D., \& LACCHINI. A. J. B. (2013). Perception of crack users in relation to use and treatment. Rev Gaúcha Enferm, 34(1), 140-146. Retrieved from: http://www.scielo.br/pdf/rgenf/v34n1/en_18.pdf

GRASSI-OLIVEIRA, R., PEZZI, J. C., DARUY-FILHO, L., VIOLA, T. W., FRANCKE, J. D., LEITE. C. E., \& BRIETZKE, E. (2012). Hair Cortisol and Stressful Life Events Retrospective Assessment in Crack Cocaine Users. The American Journal of Drug and Alcohol Abuse, 38(6), 535-538. doi:1 0.3109/00952990.2012.694538

HESS, A. R. B., ALMEIDA, R. M. M., MORAES, A. L. (2012). Comorbidades psiquiátricas em dependentes químicos em abstinência em ambiente protegido. Estudos de Psicologia,17(1), 171-178. Retrieved from: http://www.scielo.br/pdf/epsic/v17n1/21.pdf

HORTA, R. L., VIEIRA, L. S., BALBINOT, A. D., OLIVEIRA, G. O., POLETTO, S., \& TEIXEIRA, V. A. (2014). Influência da família no consumo de crack. (2014). J Bras Psiquiatr., 63 (2): 104-12. doi 10.1590/0047-2085000000013

JORGE, M. S. B., QUINDERÉ, P. H. D., YASUI, S., \& ALBUQUERQUE, R. A. (2O13). Ritual de consumo do crack: aspectos socioantropológicos e repercussões para a saúde dos usuários. Ciência \& Saúde Coletiva, 18(10), 2909-2918. doi: 1590/S1413-81232013001000015.

KASSADA, D. S., MARCON, S.S., PAGLIARINI, M. A., \& ROSSI, R. M. (2013). Prevalência do Uso de drogas de abuso POR gestantes. Acta Paulistana de Enfermagem, 26(5), 767-771. doi:10.1590/ So103-21002013000500010

KESSLER, F. (2012). Crack: personalidade antissocial é tônica entre usuários. In: Informe ENSP. Retrieved from: http://www.ensp.fiocruz.br/portal-ensp/informe/site/materia/detalhe/31006

KESSLER, F. H. P., TERRA, M. B., FALLER, S., STOLF, R. A., PEUKER, A. C. B., PECHANSKY. F. (2012) Crack users show high rates of antisocial personality disorder, engagement in illegal activities and other psychosocial problems. American Journal on Addictions, 21(4). 370-380. doi: 10.1111/j.1521-0391.2012.00245.x 
KESSLER, F., \& PECHANSKY, F. (2008). Uma visão psiquiátrica sobre o fenômeno do crack na atualidade. Revista de Psiquiatria do Rio Grande do Sul, 30(2), 96-98. doi: 10.159o/So10181082008000300003

LARANJEIRA, R. (2010). Legalização de drogas e a saúde pública. Ciência đr Saúde Coletiva, 15(3), 621-631. doi:10.1590/S1413-81232010000300002.

LIMA, H. P., MACEDO, J. Q., BRAGA, V. A. B., LEMOS, A. M., \& SILVA JÚNIOR, I. A (2011). Profile of women drug addicts treated at the Psychosocial care center alcohol and other drugs: documental study. Online Brazilian Journal Nursing, 1O(2), doi:10.5935/1676-4285.20113257

LIMBERGER, J., MELLA, L. L. DUARTE, T. O. (2014). Promoção de Saúde na Escola: o papel da Psicologia Escolar. Itinerarius Reflectionis, 10 (2). Retrieved from: http://www.revistas.ufg.br/ index.php/ritref/article/view/33046

LOPES, F., BUCHALLA, C. M., \& AYRES, J. R. C. M. (2007). Mulheres negras e não-negras e vulnerabilidade ao HIV/Aids no estado de São Paulo, Brasil. Revista de Saúde Pública, 41(Suppl.2), 39-46. Retrieved from http://www.scielo.br/scielo.php?script=sci_arttext\&pid=Soo3489102007000900008\&lng=en\&tlng=pt.\%2010.1590/So034-89102007000900008

MALTA, M., MONTEIRO, S., LIMA, R. M. J., BAUKEN, S., MARCO, A., ZUIM, G. C., ... STRATHDEE, S. A. (2008). HIV/AIDS risk among female sex workers who use crack in Southern Brazil. Rev. Saúde Pública, 42(5), 831-837. doi:10.1590/Soo34-89102008000500007

MARANGONI, S. R., \& OLIVEIRA, M. L. F. (2013). Fatores desencadeantes do uso de drogas de abuso em mulheres. Texto \& Contexto - Enfermagem, 22(3), 662-670.doi: 10.1590/So10407072013000300012.

MEDEIROS, REGINA. Construção social das drogas e do crack e as respostas institucionais e terapêuticas instituídas. Saúde e Socidade, 23(1), 105-117. doi:10.1590/So10412902014000100008

MENDES, I. A. C., \& LUIS, M. A. V., (2004). Uso de substancias psicoativas, un nuevo viejo desafío. Revista Latino-americana deEnfermagem,2, 295-6. Retrieved from: http://www.scielo.br/pdf/ rlae/v12nspe/es_v12nspeao1.pdf

NAPPO, S. A., SANCHEZ, Z., OLIVEIRA, L. G. (2011). Crack, AIDS, and Women in São Paulo, Brazil. Substance Use \& Misuse,46, 476-485. doi:10.3109/10826084.2010.503480

OLIVEIRA, J. F., \& PAIVA, M. S. (2007). Vulnerabilidade de mulheres usuárias de drogas ao HIV/ AIDS em uma perspectiva de gênero. Escola Anna Nery, 11(4), 625-631. Retrieved from: http:// www.scielo.br/scielo.php?script=sci_arttext\&pid=S1414-81452007000400011\&lng=en\&tln $\mathrm{g}=$ pt. 10.1590/S1414-81452007000400011

OLIVEIRA, J. F., PAIVA, M. S., \& VALENTE, C. M. L. (2007). A interferência do contexto assistencial na visibilidade do consumo de drogas por mulheres. Rev Latino-am Enfermagem, 15(2). Retrieved from: http://www.scielo.br/pdf/rlae/v15n2/pt_v15n2ao9.pdf 
OLIVEIRA, K. D. (2010). Perfil sóciodemográfico, padrão de consumo e comportamento riminoso em usuários de substâncias psicoativas que iniciaram tratamento [dissertação]. Campinas: Unicamp. Retrieved from: http://www.bibliotecadigital.unicamp.br/ document/?code $=000771722$

ORSI, M. M., KESSLER, F., PECHANSKY, F., ARAÚJO, R., OLIVEIRA, M. S., \& SOUZA, A. C. (2004). Características do uso de cocaína em indivíduos internados em unidades de tratamento de $\mathrm{P}$ orto Alegre, RS. J. Bras. Psiquiatr, 53(6), 351-358.

PECHANSKY, F., KESSLER, F. H. P., VON DIEMEN, L., BUMAGUIN, D. B., SURRATT, H., \& INCIARDI, J. (2007). Brazilian female crack users show elevated serum aluminum levels. Rev. Bras. Psiquiatr.29(1).39-42. doi: 10.1590/S1516-44462006005000034

PEDROSO, R. S., KESSLER, F., \& PECHANSKY, F. (2013). Treatment of female and male inpatient crack users: a qualitative study. Trends Psychiatry Psychother, 35(1), 36-45. Retrieved from http:// www.scielo.br/pdf/trends/v35n1/ao5v35n1.pdf

PINTO, V. M., TANCREDI, M. V., BUCHALLA, C. M., \& MIRANDA, A. E. (2014). History of syphilis in women living with AIDS and associated risk factors in São Paulo, Brazil. Rev Assoc Med Bras, 6o(4), 342-348. doi:10.1590/1806-9282.60.04.013

PORTELA, G. L. C., BARROS, L. M., FROTA, N. M., LANDIM, A. P. P., CAETANO, J. A, \& FARIAS, F. L. R. (2013). Perception of pregnant on consumption of illicit drugs in pregnancy. SMAD. Revista eletrônica saúde mental álcool e drogas, 9(2), 58-63. Retrieved from: http://pepsic.bvsalud. org/scielo.php?script=sci_arttext\&pid=S1806-69762013000200002\&lng=pt\&tlng=en

RAMIRO, F. S., PADOVANI, R. C., \& TUCCI, A. M. (2014). Consumo de crack a partir das perspectivas de gênero e vulnerabilidade: uma revisão sobre o fenômeno. Saúde em Debate, 38(101), 379-392. doi:10.5935/0103-1104.20140035

REY, F. L. G. (2002). Pesquisa Qualitativa em Psicologia: caminhos e desafios. São Paulo: Pioneira Thomson Learning.

RIBEIRO, M., \& LARANJEIRA, R. (2012). O tratamento do Usuário de Crack. Porto Alegre: Artmed.

RIVADENEYRA, R. E. V., CABRERA, A. P. (2010). Demora en la búsqueda de atención médica en pacientes adictos a sustancias psicoactivas. Adicciones, 22(1)73-86.

ROMANINI, M., \& ROSO, A. (2014). Midiatização do crack e estigmatização: corpos habitados por histórias e cicatrizes. Interface - Comunicação, Saúde, Educação, 18(49), 363-376. doi: 10.1590/1807-57622013.0138

ROSSETI, C. S., DORING, M., DALMOLIN, B. M., MATTOS, M., BAUMKARTEN, S. T., PETUCO, V. M., DIAS, M. T. G., \& MONTEIRO, J. R. (2014). A atenção aos usuários de crack nos leitos de saúde mental dos hospitais gerais. 32-49. In: Dalmolin, B. M., \& Doring, M. (org). Crack e outras drogas: múltiplas facetas do cuidade em saúde mental. Passo Fundo: Ed. Universidade de Passo Fundo. 
RUI, T. C. (2012). Corpos Abjetos: etnografia em cenários de uso e comércio de crack. Tese de Doutorado. Universidade Estadual de Campinas (UNICAMP). Retrieved from: http://www. neip.info/downloads/Taniele_Rui_Tese.pdf

SAKIYAMA, H. M. T., RIBEIRO, M., \& PADIN, M. F. R. (2012). Prevenção da recaída e treinamento de habilidades sociais. 337-35o. In: Ribeiro, M., Laranjeira, R. (eds.), O tratamento do usuário de crack. São Paulo: Artmed.

SAMPIERI, R.H., CALLADO, C. F., \& LUCIO, M.P. (2013). Metodologia de Pesquisa. Porto Alegre: Artmed.

SANCHÉZ, Z. M., NAPPO. S. A. (2007). A religiosidade, a espiritualidade e o consumo de drogas. Revista de Psiquiatria Clínica,34 (1).73-81. Retrieved from: http://www.hcnet.usp.br/ipq/ revista/vol34/s1/pdf/73.pdf

SAYAGO, C. B. W., LUCENA-SANTOS, P., HORTA, R. L., \& OLIVEIRA, M. S. (2014). Perfil clínico e cognitivo de usuários de crack internados. Psicol. Reflex. Crit, 27(1), 21-18. doi:10.1590/So1O279722014000100003

SELEGHIM, M. R., MARANGONI, S. R., MARCON, S. S., OLIVEIRA, M. L. F. (2O11). Vínculo familiar de usuários de crack atendidos em uma unidade de emergência psiquiátrica. Revista Latino-Americana de Enfermagem, 19(5). Retrieved from: http://www.redalyc.org/articulo. oa?id=281421964014

SELEGHIN, M. R., INOUE, K. C., SANTOS, J. A. T., \& OLIVEIRA, M. L. F. (2O11). Aspectos da estrutura familiar de jovens usuários de crack: um estudo do genograma. Ciência e Cuidado em Saúde, 10(4).795-802. doi:10.4025/cienccuidsaude.v10i4.18325

SHAUGHNESSY, J. J., ZECHMEISTER, E. B. \& ZECHMEISTER J. S., Metodologia de Pesquisa em Psicologia (2012). Porto Algre: AMGH.

SILVA, C. R., KOLLING, N. M., CARVALHO, J. C. N., CUNHA, S. M., \& KRISTENSEN, C. H. (2009). Comorbidade psiquiátrica em dependentes de cocaína/crack e alcoolistas: um estudo exploratório. Aletheia, 30, 101-112.

SODELLI, M. (2011). Drogas e Ser Humano: a prevenção do possível. 15-21. In: Conselho Regional de Psicologia da 6a Região. Álcool e outras drogas. Retrieved from: http://www.crpsp.org.br/ portal/comunicacao/livro-alcool-drogas/crpsp-alcool-e-outras-drogas.pdfs.

SOUZA, M. R. R., OLIVEIRA, J. F., \& NASCIMENTO, E. R. (2014). A saúde de mulheres e o fenômeno das drogas em revistas brasileiras. Texto Contexto Enferm. 23(1), 92-10o. Retrieved from: http://www.redalyc.org/pdf/714/71430666o11.pdf

TRACTENBERG, S. G., VIOLA, T. W., ROSA, C. S. O., DONATI, J. M., FRANCKE, I. A., PEZZI, J. C., \& GRASSI-OLIVEIRA, R. Exposição a trauma e transtorno de estresse pós-traumático em usuárias de crack. Jornal Brasileiro de Psiquiatria, 61(4), 206-213. doi: 10.1590/Soo4720852012000400003 
TURATO, E. R. (2005). Métodos qualitativos e quantitativos na área da saúde: definições, diferenças e seus objetos de pesquisa. Revista de Saúde Pública, 39 (3): 507-514.

VELÁSQUEZ, M., MAURER, G., CROUCH, C., \& DICLEMENTE, C. (2001) Group treatment for substance abuse: a stages-of-change therapy manual. New York: The Guilford Press.

VIOLA, T. W., TRACTENBERG, S. G., PEZZI, J. C., KRISTENSEN, C. H., \& GRASSI-OLIVEIRA,R. (2013). Childhood physical neglect associated with executive functions impairments in crack cocaine-dependent women. Drug and Alcohol Dependence, 132, 271-272. doi: 10.1016/j. drugalcdep.2013.02.014

VON DIEMEN, L., DE BONI, R., KESSLER, F., BENZANO, D., \& PECHANSKY, F. (2O10). Risk behaviors for HCV- and HIV-seroprevalence among female crack users in Porto Alegre, Brazil. Arch Womens Ment Health, 13(3), 185-191. doi: 10.1007/soo737-009-0089-y

WECHSLER, D. (2012). WAIS-III: Escala de Inteligência Wechsler para Adultos: manual de administração e avaliação. São Paulo: Casa do Psicólogo.

WOLLE, C. C., ZIBERMAN, M. L. (2011). Mulheres.375-382. In: Diehl A; Cordeiro, D. C. \& Laranjeira, R. (Org.). Dependência Química:prevenção, tratamento e políticas públicas, Porto Alegre: Artmed,

YIN, R. K. (2001). Estudo de caso: planejamento e métodos. 2. ed. Porto Alegre: Bookman. 